AperTO - Archivio Istituzionale Open Access dell'Università di Torino

\title{
Evolutionary Algorithm-based Crystal Structure Prediction for Copper(I) Fluoride
}

\section{This is the author's manuscript}

Original Citation:

Availability:

This version is available http://hdl.handle.net/2318/1766416

since 2021-01-12T11:54:53Z

Published version:

DOI:10.1002/chem.201902314

Terms of use:

Open Access

Anyone can freely access the full text of works made available as "Open Access". Works made available under a Creative Commons license can be used according to the terms and conditions of said license. Use of all other works requires consent of the right holder (author or publisher) if not exempted from copyright protection by the applicable law. 


\section{CHEMISTRY A European Journal}

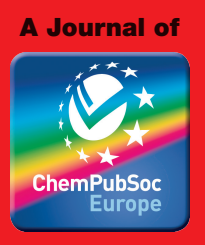

\section{Accepted Article}

Title: Evolutionary Algorithm-based Crystal Structure Prediction for Copper(I) Fluoride

Authors: Mikhail S. Kuklin, Lorenzo Maschio, Denis Usvyat, Florian Kraus, and Antti J. Karttunen

This manuscript has been accepted after peer review and appears as an Accepted Article online prior to editing, proofing, and formal publication of the final Version of Record (VoR). This work is currently citable by using the Digital Object Identifier (DOI) given below. The VoR will be published online in Early View as soon as possible and may be different to this Accepted Article as a result of editing. Readers should obtain the VoR from the journal website shown below when it is published to ensure accuracy of information. The authors are responsible for the content of this Accepted Article.

To be cited as: Chem. Eur. J. 10.1002/chem.201902314

Link to VoR: http://dx.doi.org/10.1002/chem.201902314 


\title{
Evolutionary Algorithm-based Crystal Structure Prediction for Copper(I) Fluoride
}

\author{
Mikhail S. Kuklin, ${ }^{[a]}$ Lorenzo Maschio, ${ }^{[b]}$ Denis Usvyat, ${ }^{[c]}$ Florian Kraus, ${ }^{[d]}$ Antti J. Karttunen ${ }^{*}\left[{ }^{[a]}\right.$
}

\begin{abstract}
Despite numerous experimental studies since 1824, the binary copper(I) fluoride still remains unknown. We have carried out a crystal structure prediction for CuF using the USPEX evolutionary algorithm and a dispersion-corrected hybrid density functional method. In total about 5000 hypothetical structures were investigated. The energetics of the predicted structures were also counter-checked with local second-order Møller-Plesset perturbation theory. We report 39 new hypothetical copper(I) fluoride structures that are lower in energy compared to the previously predicted cinnabar-type structure. Cuprophilic $\mathrm{Cu}-\mathrm{Cu}$ interactions are present in all the low-energy structures, leading to ordered $\mathrm{Cu}$ substructures such as helical or zig-zag-type $\mathrm{Cu}-\mathrm{Cu}$ motifs. The lowest-energy structure adopts a trigonal crystal structure with space group $P 3_{1} 21$. From the electronic point of view, the predicted CuF modification is a semiconductor with an indirect band gap of $2.3 \mathrm{eV}$.
\end{abstract}

\section{Introduction}

Binary copper(I) halides $\mathrm{CuCl}, \mathrm{CuBr}$, and $\mathrm{Cul}$ are known to be semiconductors possessing the cubic zincblende structure (space group F-43m). ${ }^{1}$ In contrast, copper(I) fluoride (CuF) has not been synthesized unambiguously and is not structurally characterized to this date. In the case of CuF, a major challenge is the disproportionation of $\mathrm{Cu}(\mathrm{I})$ to $\mathrm{Cu}(\mathrm{II})$ and $\mathrm{Cu}$ metal in aqueous solution: $\mathrm{CuF} \rightarrow \mathrm{CuF}_{2}+\mathrm{Cu}$. The first report on the synthesis of CuF dates back to Berzelius in 1824, and since then a series of attempts have been made to obtain $\mathrm{CuF}^{2-7}$ However, to date there has not been a reproducible successful synthesis of CuF and its structure remains a mystery. ${ }^{5,8-11}$ An early experimental attempt to describe the crystal structure of CuF was made in $1933,{ }^{3}$ suggesting that CuF possesses the

[a] Dr. Mikhail Kuklin, Prof. Antti J. Karttunen Department of Chemistry and Materials Science Aalto University 00076 Aalto, Finland E-mail: antti.j.karttunen@iki.fi

[b] Prof. Lorenzo Maschio

Dipartimento di Chimica, C3S Centere, NIS Centre Università di Torino

Via P. Giuria 5, 10125 Torino, Italy

[c] Dr. Denis Usvyat

Institut für Chemie

Humboldt Universität zu Berlin

Brook-Taylor-Str. 2, D-12489 Berlin, Germany

[d] Prof. Dr. Florian Kraus

Fachbereich Chemie

Philipps-Universität Marburg

Hans-Meerwein-Strasse 4, 35032, Marburg, Germany

Supporting information for this article is given via a link at the end of the document. same zincblende structure as the other $\mathrm{Cu}(\mathrm{I})$ halides. This observation is still being reported in some textbooks and crystal structure databases even though Haendler et al. later showed that the interplanar spacings of the CuF crystal structure reported in 1933 are practically identical to those in $\mathrm{Cu}_{2} \mathrm{O} .{ }^{10}$ In several other studies, the characterization of the product assumed to be CuF was not possible due to the disproportionation to $\mathrm{CuF}_{2}$ and $\mathrm{Cu}_{4}{ }^{4,5,12}$

Due to the experimental challenges in the synthesis of CuF, several theoretical and computational studies towards improved understanding of $\mathrm{CuF}$ and its structural chemistry have been carried out. Barber et al. calculated lattice energies for monofluorides of the $3 d$ metals using simple empirical formula and reported all of them to be unstable towards disproportionation. ${ }^{13}$ Schwerdtfeger et al. carried out pioneering computational studies on the possible structure of CuF by comparing rocksalt, zincblende, and cluster models using density functional theory (DFT). ${ }^{14,15}$ Walsh et al. used DFT methods to investigate six hypothetical CuF structures based on known binary structure types: cinnabar, graphite, NiAs, sphalerite, rocksalt, and wurtzite. ${ }^{16}$ They identified cinnabar as the lowest-energy structure for CuF by using a hybrid DFT method (Figure 1a). The structures of the binary compounds investigated by Walsh et al. were also included in a later study where 22 hypothetical CuF structures were investigated using hybrid DFT and Local-MP2 (LMP2) methods. ${ }^{11}$ Also in this study, the cinnabar structure was found to be the lowest-energy structure for CuF. Besides the cinnabar structure, a low-energy structure derived by distorting a wurtzite-type structure was found (Figure 1b). (a)

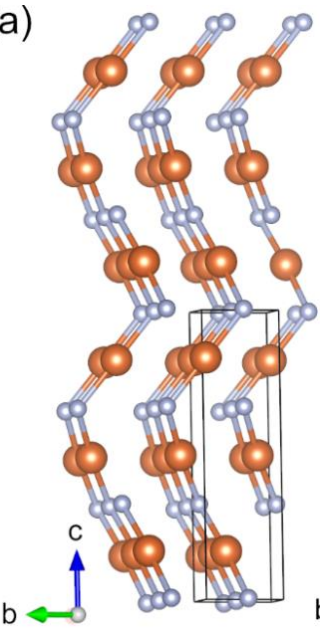

(b)

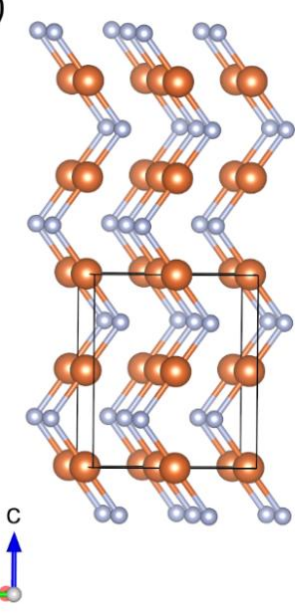

Figure 1. The lowest-energy crystal structures of CuF identified in previous computational studies: (a) cinnabar-type structure $\left(P 3_{1} 21\right)^{16}$ and (b) distorted wurtzite-type structure $(\mathrm{Cmcm}) .{ }^{11}$ Reddish brown: $\mathrm{Cu}$, light blue: $\mathrm{F}$. 
So far, all hypothetical CuF crystal structures considered in computational studies have been derived from known binary structure types, which is a very strong limitation on the search space. To account for the possibility that CuF adopts its own new structure type, one may utilize crystal structure prediction algorithms that enable screening of a vast number of hypothetical structures. ${ }^{17-21}$ Evolutionary algorithm-based methods (EA) have been proved to be one of the most successful techniques to search for global minima and to predict unknown crystal structures. ${ }^{22-27}$ An example of a robust EAbased method is the USPEX code (Universal Structure Predictor: Evolutionary Xtallography), which has been successfully used for numerous different materials. ${ }^{24-33}$

Here, we describe the first evolutionary algorithm-based crystal structure prediction study for copper(I) fluoride. By combining the USPEX method with dispersion-corrected hybrid DFT method and ab initio LMP2 calculations, we are able to report 39 hypothetical CuF structures that have lower energy than the previously reported cinnabar-type structure.

\section{Results and Discussion}

\section{Overview of the CuF structures predicted by USPEX.}

To span the configuration space of an unknown crystal structure such as CuF, different numbers of formula units $(Z)$ have to be considered. For $\mathrm{CuF}$, we investigated compositions from $Z=2$ to $Z=8$. For each case, except for $Z=7$ and $Z=8$, we ran several distinct USPEX searches to improve the sampling of the configuration space (see below for details). In some cases, a symmetry analysis of the structural candidate obtained from USPEX resulted in a final structure with smaller $Z$ than in the original USPEX search. For example, an USPEX search with $Z$ $=2, Z=4, Z=6, Z=8$ could yield a final structure with $Z=1, Z$
$=2, Z=3$, or $Z=4$ respectively. We did not run USPEX for $Z=1$ as this composition proved to be too small in our tests. Furthermore, the simulations with $Z=2-8$ yielded many final structures with $Z=1$, which were found to be high-energy structures not discussed here. In general, the number of formula units used in the USPEX simulations is limited by the available computational resources and searches with $Z=7$ and $Z=8$ proved already to be rather demanding with the hybrid DFTPBE0 method.

As the cinnabar-type structure was previously obtained as the lowest-energy crystal structure for CuF, we used it as a reference for our energy comparisons. Thus, all relative energies $(\Delta E)$ are reported with respect to the cinnabar-type $(Z=3)$ :

$\Delta E=E(\mathrm{CuF}$ structure $) / Z(\mathrm{CuF}$ structure $)-E$ (cinnabar-type $\mathrm{CuF}) / Z$ (cinnabar-type CuF).

Figure 2 illustrates the relative energies of the predicted lowest-energy CuF structures and Table 1 lists detailed information for them. The structures are labeled using a scheme that is explained in the caption of Figure 2. The found structures as well as the differences between the DFT-PBE0-D3/TZVP and LMP2/TZVPP levels of theory are discussed in more detail in the following sections. The unit cell parameters and atom coordinates of the predicted CuF structures are given as $\mathrm{SI}$ in CIF format.

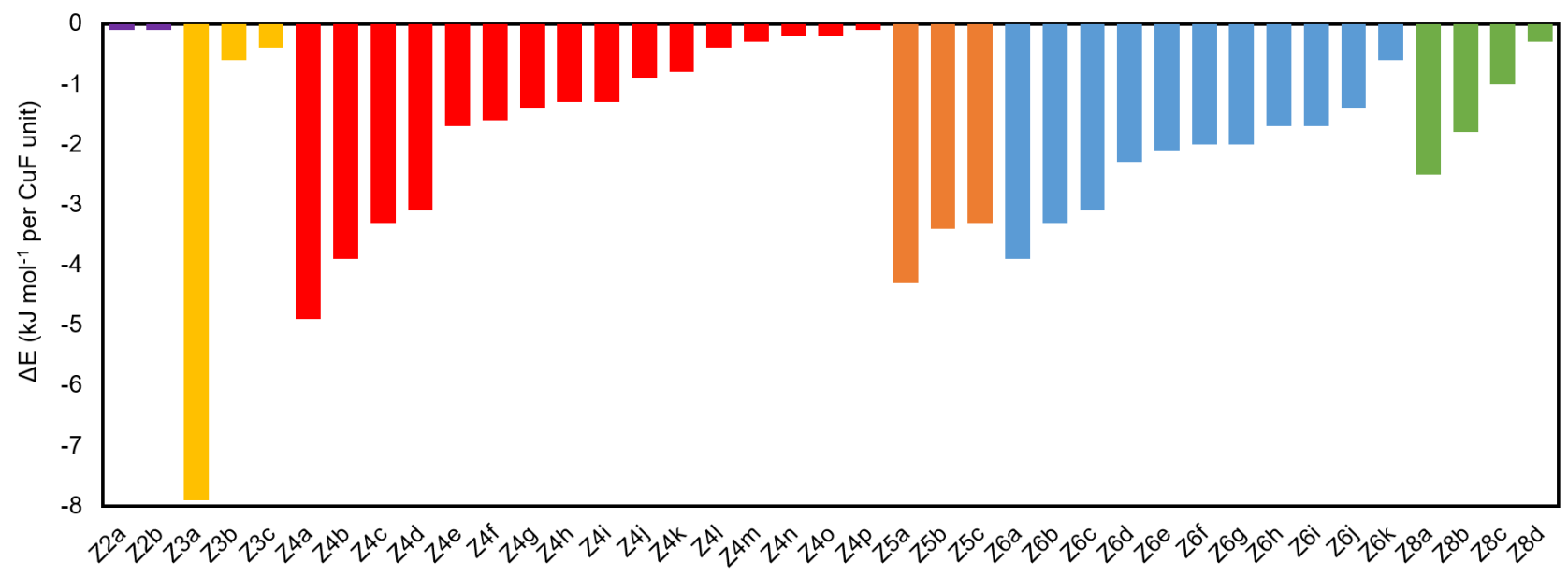

Figure 2. Relative DFT-PBE0-D3/TZVP energies of the lowest-energy CuF structures predicted by USPEX. The energies are given with respect to the cinnabar-type CuF $(\Delta E=0)$. The structures are identified by a label like Z2a. The label tells the number of formula units used in the USPEX search that produced the structure $(Z=2$ for $\mathbf{Z 2 a})$. The letter $(\mathbf{a}, \mathbf{b}, \mathbf{c}, \ldots)$ tells the energy ranking of the structure within this class of structures, $\mathbf{a}$ being the lowest-energy structure. Note that the final $Z$ may be different from the $Z$ used in the original USPEX simulation (see Table 1). 
Table 1. Space group, formula units (Z), relative energy, band gap, density, and bonding situation for the lowest-energy CuF structures predicted in this study.

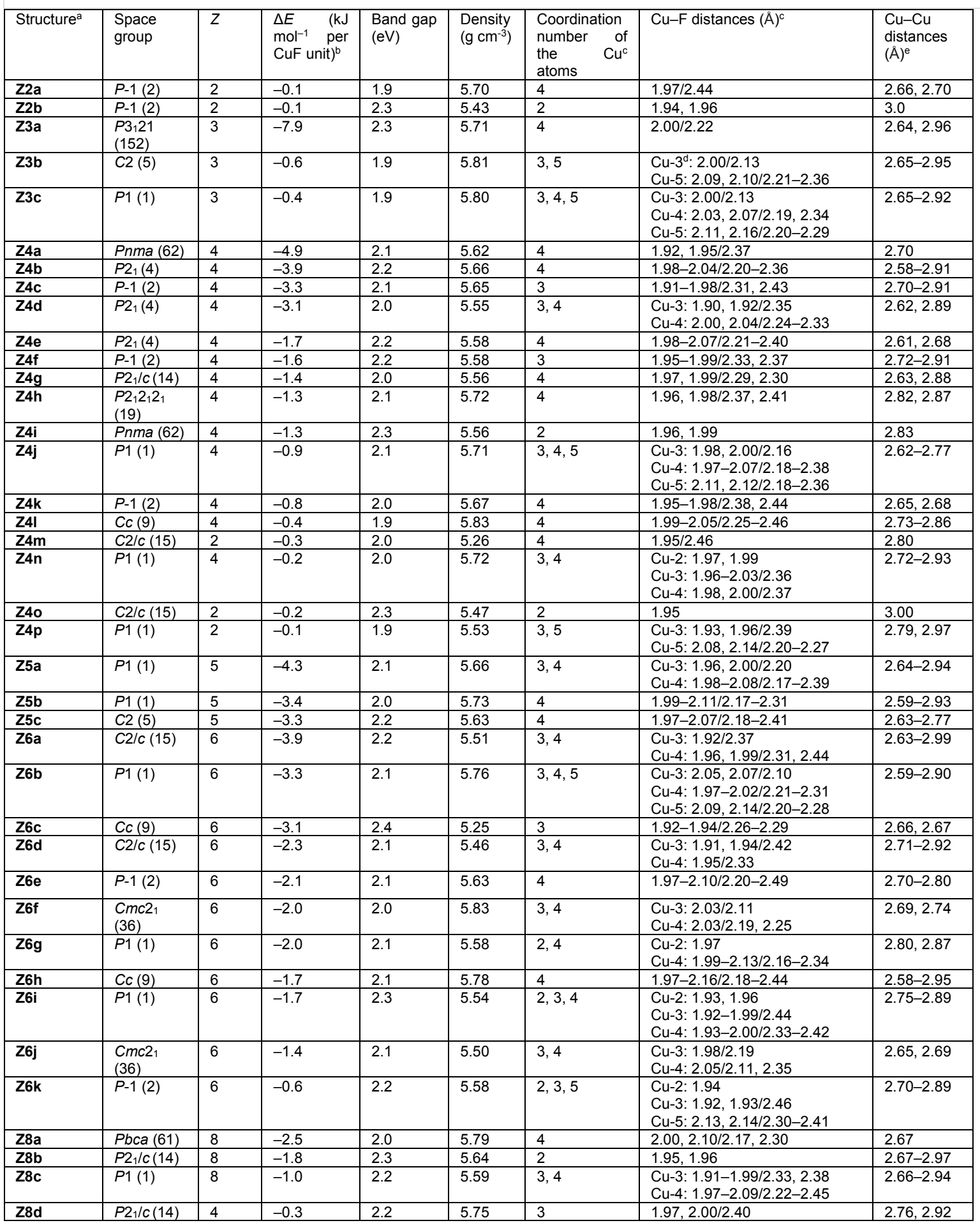

${ }^{a}$ Label of the structure. See caption of Figure 2 for an explanation of the labeling.

b Relative energy compared to the previously reported cinnabar-type CuF.

c $\mathrm{Cu}-\mathrm{F}$ distances smaller than $2.49 \AA$ were used to determine the coordination number of $\mathrm{Cu}$.

${ }^{d}$ When there are $\mathrm{Cu}$ atoms with different coordination numbers, these are denoted as $\mathrm{Cu}-3, \mathrm{Cu}-4$, etc.

e $\mathrm{Cu}-\mathrm{Cu}$ distances longer than $3 \AA$ are not listed. 


\section{CuF structures from USPEX simulations with composition $\mathrm{Cu}_{2} \mathrm{~F}_{2}$}

We started the structure prediction with a composition possessing only two copper and two fluorine atoms in the unit cell (Cu2F2). Overall, five USPEX simulations were carried out, screening 1090 hypothetical Cu2F2 structures in total. However, only two structures were found to possess relative energy that is similar to the cinnabar-type structure: Z2a (space group $P$-1) and Z2b (space group $P-1$ ). Both structures are triclinic, whereas the cinnabar-type CuF should adopt the trigonal space group P3121. Band gaps of Z2a and Z2 $\mathbf{b}$ are rather similar at about $2 \mathrm{eV}$, while the density of the $\mathbf{Z 2 a}$ structure $(5.70 \mathrm{~g} \mathrm{~cm}-3)$ is larger than that of $\mathbf{Z 2 b}(5.43 \mathrm{~g} \mathrm{~cm}-3)$.

The USPEX simulation for $Z=2$ also produced the distorted wurtzite structure discovered in a previous study to be a lowenergy structure (Figure 1b). ${ }^{11}$ Compared to the previous PBE0/TZVP results, we found that taking dispersion interactions into account clearly changes the energetics of the distorted wurtzite structure. Previously the structure was estimated to be $1.2 \mathrm{~kJ} \mathrm{~mol}^{-1}$ per CuF unit higher in energy than the cinnabartype structure, but inclusion of the dispersion corrections increases the relative energy to $2.8 \mathrm{~kJ} \mathrm{~mol}^{-1}$. Structural parameters of the distorted wurtzite also change clearly in case of DFT-PBE0-D3/TZVP, resulting in $a=2.90 \AA, b=5.38 \AA$, and $c=6.25 \AA$, while the corresponding DFT-PBE0/TZVP values were $3.09 \AA, 5.50 \AA$, and $6.38 \AA$. Cu-F distances are almost identical, $1.95 \AA$ and $1.96 \AA$, for DFT-PBE0/TZVP and DFTPBE0-D3/TZVP, respectively.

\section{CuF structures from USPEX simulations with composition $\mathrm{Cu}_{3} \mathrm{~F}_{3}$}

We ran three USPEX simulations with composition $\mathrm{Cu}_{3} \mathrm{~F}_{3}$, resulting in total in 591 hypothetical CuF structures. Three structures were found to possess lower energy than the cinnabar-type structure (Table 3 ). All $Z=3$ structures produced by USPEX are lower in energy than the structures obtained with the starting composition $\mathrm{Cu}_{2} \mathrm{~F}_{2}$. Among all predicted $\mathrm{CuF}$ structures, structure Z3a was found to be the lowest-energy structure with $\Delta E=-7.9 \mathrm{~kJ} \mathrm{~mol}^{-1}$ per CuF.

Z3a adopts a trigonal crystal structure with space group $P 3_{1} 21$. This is in fact the same space group as for the cinnabar-type $\mathrm{CuF}$ and the structure is closely related (Figure 3 ) as also the Pearson code and Wyckoff sequence are similar. If only the shortest $\mathrm{Cu}-\mathrm{F}$ distances are considered $(2.0 \AA)$, the coordination number of both the $\mathrm{Cu}$ and $\mathrm{F}$ atoms is two. While the $\mathrm{Cu}$ atom is almost linearly coordinated by two $\mathrm{F}$ atoms $\left(162.5^{\circ}\right)$, the $\mathrm{F}$ atom interconnects two $\mathrm{Cu}$ atoms in an angle of $82.3^{\circ}$. The $3_{1}$ screw axis of the space group leads to onedimensional infinite helices described with the Niggli formula ${ }_{1}\left[\mathrm{Cu}_{2 / 2} \mathrm{~F}_{2 / 2}\right]$ parallel to the $c$-axis as is the case for $\mathrm{\alpha}-\mathrm{HgS}$. Due to the $3_{1}$ screw axis, one turn of the helix is finished after the length of the $c$-axis. One turn of the $\mathrm{Cu}-\mathrm{F}$-helix is finished after the $c$-axis length of $2.96 \AA$, whereas in $\alpha-\mathrm{HgS}$ one turn is finished after circa $9.44 \AA$. While the coordination numbers of the $\mathrm{Hg}$ and $\mathrm{S}$ atoms in $\alpha-\mathrm{HgS}$ can be described as $\mathrm{Hg}^{[2+2+2]} S^{[2+2+2]}$, both reminiscent of octahedra as in galena $(\mathrm{PbS})$, the coordination numbers of the respective atoms in
CuF are much better described as $\mathrm{Cu}^{[2+2]} \mathrm{F}^{[2+2]}$. In $\alpha-\mathrm{HgS}$, the $\mathrm{Hg}-\mathrm{S}$ distances within the octahedron-like coordination polyhedron are $2.2,3.1$, and $3.5 \AA$. The respective $\mathrm{Cu}-\mathrm{F}$ distances are 2.0 and $2.2 \AA$, while the next-nearest $\mathrm{Cu}-\mathrm{F}$ distances are $3.1 \AA$. So, a coordination number of 4 in $\mathrm{CuF}$ is much more pronounced in comparison to $\mathrm{a}-\mathrm{HgS}$.

Based on single-bond covalent radii from the literature, $\mathrm{Cu}-\mathrm{F}$ single bonds would be expected to be about $1.76 \AA .{ }^{63}$ However, in $\mathrm{CuF}_{2}$ with a Jahn-Teller distorted octahedral coordination, there are four $\mathrm{Cu}-\mathrm{F}$ distances of $1.91 \AA$ and two distances of $2.31 \AA$. The CuF structures predicted here have $\mathrm{Cu}-\mathrm{F}$ distances that are rather similar to $\mathrm{CuF}_{2}$, a typical pattern being that there are two distances slightly below $2.0 \AA$ and two distances clearly longer than this (about 2.2-2.4 $\AA$ ). The coordination numbers in Table 1 have been reported in such way that both the "short" and "long" $\mathrm{Cu}-\mathrm{F}$ distances are counted in the coordination number.

Table 1 shows that the predicted $\mathrm{CuF}$ structures have $\mathrm{Cu}-\mathrm{Cu}$ distances shorter than $3.0 \AA$, suggesting weak cuprophilic $\mathrm{Cu}-$ $\mathrm{Cu}$ interactions. The shortest $\mathrm{Cu}-\mathrm{Cu}$ distance in Z3a is $2.64 \AA$. Each $\mathrm{Cu}$ atom has two $\mathrm{Cu}-\mathrm{Cu}$ distances of $2.64 \AA$ and two longer $\mathrm{Cu}-\mathrm{Cu}$ distances of $2.96 \AA$ that are equal to the lattice parameter $c$. Due to the $3{ }_{1}$ screw axis, also the closer $\mathrm{Cu}-\mathrm{Cu}$ contacts form helical chains along the $c$-axis (Figure $3 b$ ). The density and band gap of Z3a are $5.71 \mathrm{~g} \mathrm{~cm}^{-3}$ and $2.3 \mathrm{eV}$, respectively. The band gap is indirect and the valence bands are dominated by contributions from $\mathrm{Cu}$ atoms. The band structure and the density of states of Z3a are illustrated in the SI (Figure S1). 
(a)

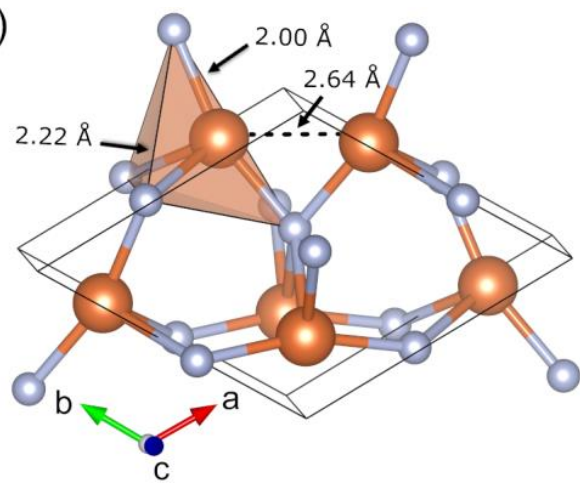

(b)

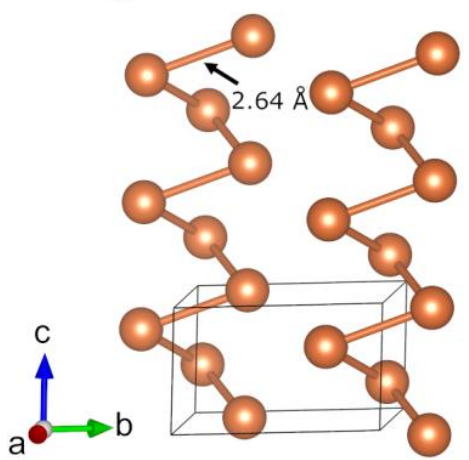

(c)

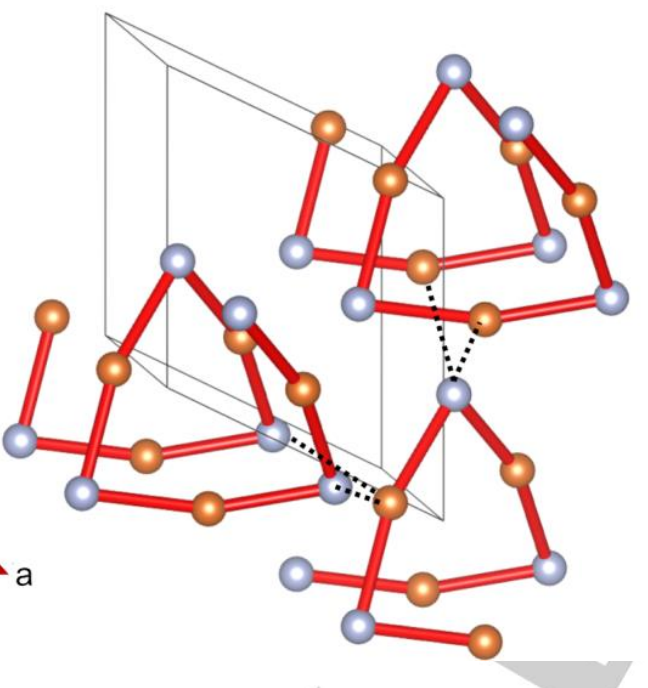

Figure 3. Predicted low-energy CuF structure Z3a $\left(P 3_{1} 21, Z=3\right)$. Reddish brown atoms are $\mathrm{Cu}$, light blue atoms $\mathrm{F}$. a) Primitive unit cell, b) Illustration of the $\mathrm{Cu}$ atom substructure and the short $\mathrm{Cu}-\mathrm{Cu}$ distances of $2.64 \AA$, c) View that highlights the $\mathrm{Cu}-\mathrm{F}$ helices. $\mathrm{Cu}-\mathrm{F}$ distances of $2.0 \AA$ shown in red and the interhelical $\mathrm{Cu}-\mathrm{F}$ distances of $2.2 \AA$ shown as dotted lines.

\section{CuF structures from USPEX simulations with composition $\mathrm{Cu}_{4} \mathrm{~F}_{4}$}

We carried out five USPEX simulations with composition $\mathrm{Cu}_{4} \mathrm{~F}_{4}$, resulting in total in 1441 hypothetical CuF structures. 16 structures were found to possess lower energy than the cinnabar-type structure (Table 1). Three of the low-energy structures obtained from a starting composition $\mathrm{Cu}_{4} \mathrm{~F}_{4}$ actually have $Z=2$ in the final crystal structure (Z4m, Z4o, and Z4p).
As in case of $Z=3$, the lowest-energy $Z=4$ structures produced by the USPEX simulation with the starting composition $\mathrm{Cu}_{4} \mathrm{~F}_{4}$ are lower in energy than the structures obtained with starting composition $\mathrm{Cu}_{2} \mathrm{~F}_{2}$, indicating that $Z=2$ is not large enough to cover the CuF configuration space. Among all predicted CuF structures with $Z=4$, structure Z4a was found to be the lowest-energy structure with $\Delta E=-4.9 \mathrm{~kJ}$ $\mathrm{mol}^{-1}$ per CuF (Figure 4).

(a)

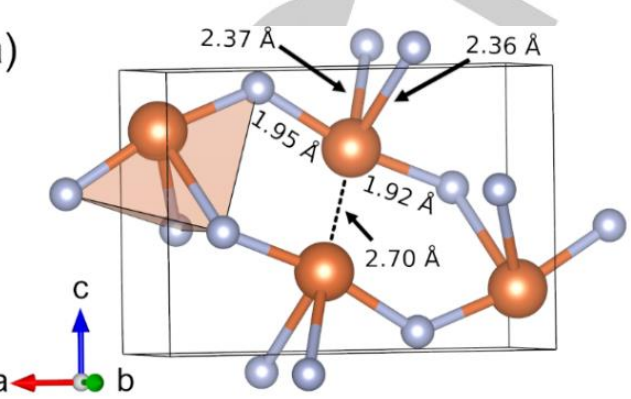

(b)

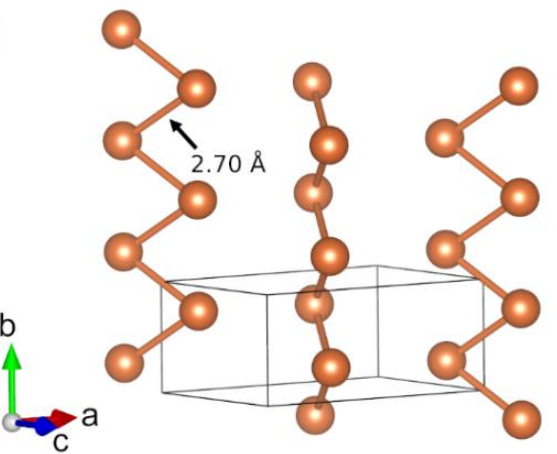

(c)

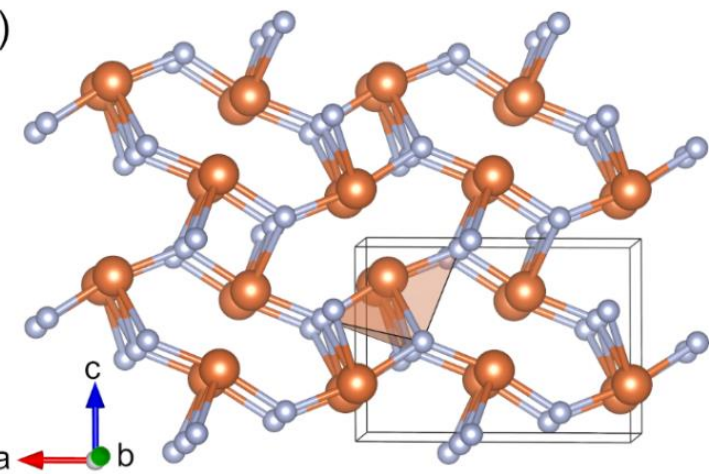

Figure 4. Predicted low-energy CuF structure Z4a (Pnma, $Z=4)$. Reddish brown atoms are $\mathrm{Cu}$, light blue atoms $\mathrm{F}$. a) Primitive unit cell, b) Illustration of the $\mathrm{Cu}$ atom substructure and the short $\mathrm{Cu}-\mathrm{Cu}$ distances of $2.70 \AA, \mathrm{c}$ ) $2 \times 2 \times 2$ supercell of the structure.

The structure Z4a adopts an orthorhombic crystal structure with space group Pnma. The coordination number of both $\mathrm{Cu}$ and $\mathrm{F}$ atoms in $\mathbf{Z 4 a}$ is 4 , with two shorter $\mathrm{Cu}-\mathrm{F}$ distances of 1.92 and $1.95 \AA$ and two longer distances of 2.36 and $2.37 \AA$ (Figure $4 a$ ). The $\mathrm{F}-\mathrm{Cu}-\mathrm{F}$ angle is $168.9^{\circ}$ for the shorter and $76.0^{\circ}$ for the longer $\mathrm{Cu}-\mathrm{F}$ distances, respectively. Each $\mathrm{Cu}$ atom has two short $\mathrm{Cu}-\mathrm{Cu}$ distances of $2.70 \AA$, forming a zig- 
zag-type chain of such interactions along the $b$-axis of the structure (Figure $4 b$ ). The $\mathrm{Cu}-\mathrm{Cu}$ distance in Z3a is slightly shorter $(2.64 \AA)$. Each $\mathrm{Cu}$ atom also possesses two nextnearest neighbors at a $\mathrm{Cu}-\mathrm{Cu}$ distance of $2.91 \AA$ along the $b$ axis. The band gap of about $2 \mathrm{eV}$ and the density of about 5.6 $\mathrm{g} \mathrm{cm}^{-3}$ for the Z4a structure are rather typical among the Z4 structures.

Overall, as in the case of the $\mathbf{Z 2}$ and $\mathbf{Z 3}$ structures, the $\mathbf{C u}-$ $F$ distances in $\mathbf{Z 4}$ structures are about 1.9-2.0 $\AA$ for the shorter and about 2.2-2.4 $\AA$ for the longer ones. The $\mathrm{Cu}-\mathrm{Cu}$ distances vary from 2.6 to $2.9 \AA$, the only exception being $Z 40$ with $3.0 \AA$. The coordination numbers of $\mathbf{C u}$ in the $\mathbf{Z 4}$ structures vary from 2 to 5 and some structures possess $\mathrm{Cu}$ atoms with different coordination numbers (Z4d, Z4j, Z4n, and Z4p).

Besides the structure $\mathbf{Z 4 a}$, the structures $\mathbf{Z 4 b}\left(P 2_{1}\right), \mathbf{Z 4} \mathbf{c}$ $(P-1)$, and $\mathbf{Z} 4 \mathbf{d}\left(P 2_{1}\right)$ were found to be low-energy structures with $\Delta E$ between -3 and $-4 \mathrm{~kJ} \mathrm{~mol}^{-1}$ (Figure 5). As the structures have rather low symmetry, they possess a range of different $\mathrm{Cu}-\mathrm{F}$ distances, which are overall rather similar: 1.9$2.0 \AA$ for the short distances and 2.2-2.4 $\AA$ for the long distances. $\mathrm{Cu}-\mathrm{Cu}$ distances vary between 2.6 and $2.9 \AA$.
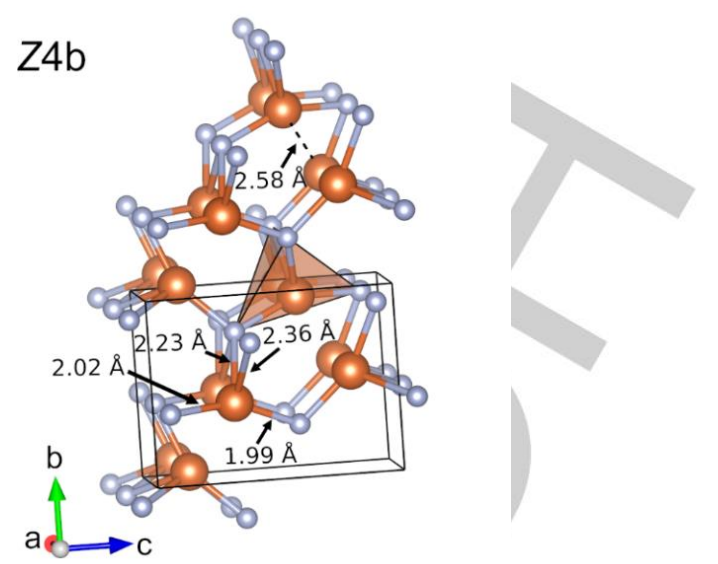

Z4C
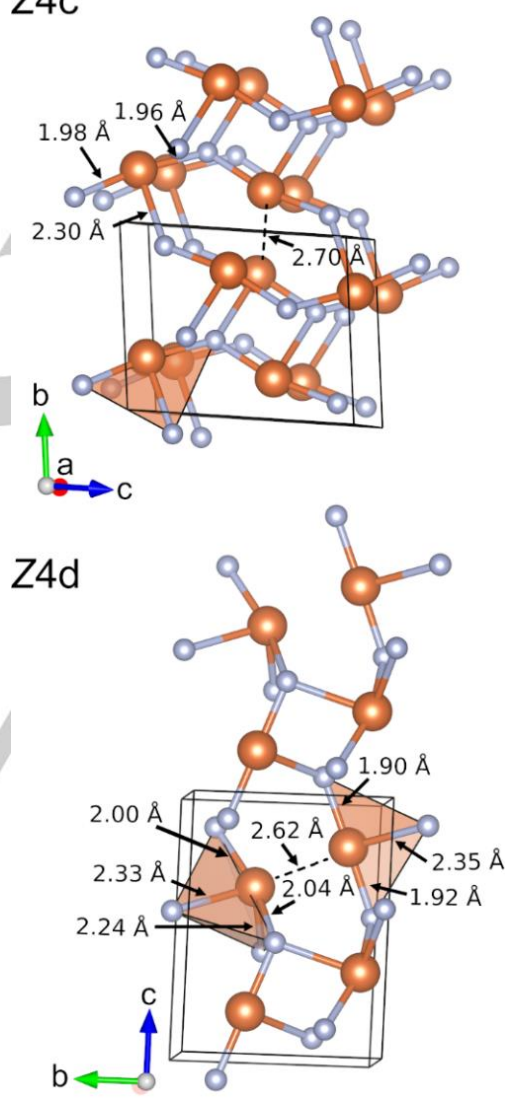

Figure 5. Low-energy CuF structures Z4b, Z4c, and Z4d, obtained from USPEX simulations with composition $\mathrm{Cu}_{4} \mathrm{~F}_{4}$. Reddish brown atoms are $\mathrm{Cu}$, light blue atoms $\mathrm{F}$.

Other Z4 structures that are not illustrated here are available as $\mathrm{SI}$ in $\mathrm{CIF}$ format. One of the predicted $\mathbf{Z 4}$ structures, $\mathbf{Z 4 g}\left(P 2_{1} / \mathrm{c}\right)$, looks similar to the distorted $\mathrm{MnP}$ structure investigated also in a previous study on hypothetical CuF structures. ${ }^{11}$ Also, structures Z4a $(P n m a), \mathbf{Z 4 d}\left(P 2_{1}\right), \mathbf{Z 4 g}$ $\left(P 2_{1} / c\right)$, and $\mathbf{Z 4 k}(P-1)$ seem to have a very similar framework; however, the energy difference between $\mathbf{Z 4 a}$ and $\mathbf{Z 4 k}$ is $4.1 \mathrm{~kJ}$ 
$\mathrm{mol}^{-1}$ per CuF unit (Table 1). This illustrates that even small structural modifications lead to significant changes in the energetics of the CuF structures studied here.

\section{CuF structures from USPEX simulations with composition $\mathrm{Cu}_{5} \mathrm{~F}_{5}$}

We carried out two USPEX simulations with composition $\mathrm{Cu}_{5} \mathrm{~F}_{5}$, producing 440 hypothetical structures. Only three predicted $Z=5$ structures were found to have lower energy than the cinnabar-type structure (Table 1). However, all of them possess rather low relative energy (Figure 6). All $Z=5$ candidates are low-symmetry structures, Z5a and Z5b being triclinic and Z5c monoclinic (C2). Z5a includes two types of Cu atoms with different coordination numbers (three and four), whereas all $\mathbf{C u}$ atoms in $\mathbf{Z 5 b}$ and $\mathbf{Z 5 c}$ are surrounded by four fluorine atoms. The $\mathrm{Cu}$ substructure in $\mathbf{Z 5 a}$ combines helical and zig-zag motifs seen in $\mathbf{Z} 3$ and $\mathbf{Z} \mathbf{2}$ structures.
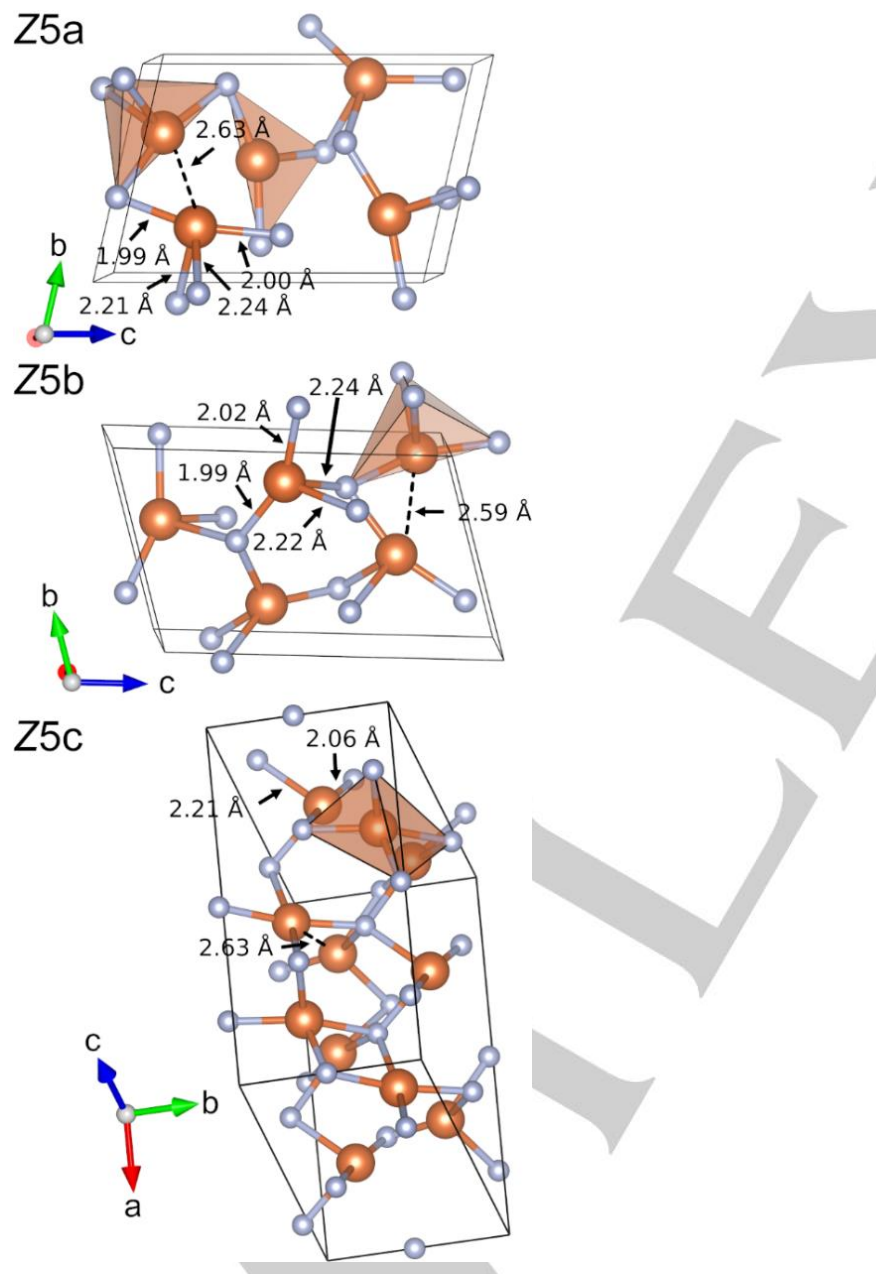

Figure 6. Low-energy CuF structures Z5a, Z5b, and Z5c, obtained from USPEX simulations with composition $\mathrm{Cu}_{5} \mathrm{~F}_{5}$. Reddish brown atoms are $\mathrm{Cu}$, light blue atoms $\mathrm{F}$.

\section{CuF structures from USPEX simulations with composition $\mathrm{Cu}_{6} \mathrm{~F}_{6}$}

We ran three USPEX simulations with composition $\mathrm{Cu}_{6} \mathrm{~F}_{6}$, resulting in total 1068 structures and 11 hypothetical structures that have lower energy than cinnabar-type CuF (Table 1). The USPEX simulation with composition $\mathrm{Cu}_{6} \mathrm{~F}_{6}$ also reproduced the lowest-energy structure Z3a, but here we focus on structures with $Z=6$. The structures Z6a (C2/c), Z6b (P1), and Z6c (Cc) were found to be among the lowest-energy hypothetical CuF structures (Figure 7), their relative energies being rather similar to structures Z4b, Z4c, and Z4d (Z6a: $-3.9 \mathrm{~kJ} \mathrm{~mol}^{-1}$, Z6b: -3.3 $\left.\mathrm{kJ} \mathrm{mol}{ }^{-1}, \mathbf{Z 6 c}:-3.1 \mathrm{~kJ} \mathrm{~mol}^{-1}\right)$. The structure $\mathbf{Z 6 c}$ has the lowest density among all studied CuF structures $\left(5.25 \mathrm{~g} \mathrm{~cm}^{-3}\right)$. Cu-F and $\mathrm{Cu}-\mathrm{Cu}$ distances of all $\mathbf{Z 6}$ structures are rather similar: short $\mathrm{Cu}-\mathrm{F}$ distances are about $2.0 \AA$, and longer distances are about 2.2-2.4 $\AA$. $\mathrm{Cu}-\mathrm{Cu}$ distances are about 2.7-3.0 $\AA$. These ranges are also similar to $\mathbf{Z 2}$ and $\mathbf{Z 4}$ structures.

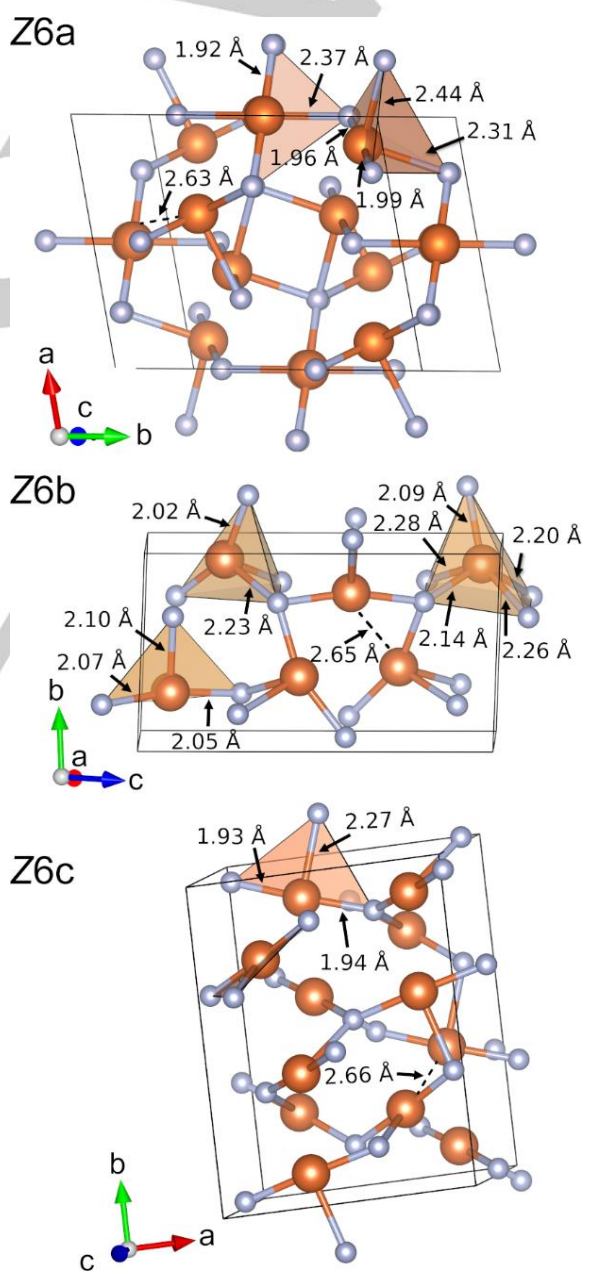

Figure 7. Low-energy CuF structures Z6a, Z6b, and Z6c, obtained from USPEX simulations with composition $\mathrm{Cu}_{6} \mathrm{~F}_{6}$. Reddish brown atoms are $\mathrm{Cu}$, light blue atoms $\mathrm{F}$. 
CuF structures from USPEX simulations with composition $\mathrm{Cu}_{7} \mathrm{~F}_{7}$

We ran one USPEX simulation with the composition $\mathrm{Cu}_{7} \mathrm{~F}_{7}$, producing 231 structures in total. No structures were found to possess lower energy in comparison to cinnabar-type CuF. The lowest energy $\mathrm{Cu}_{7} \mathrm{~F}_{7}$ structure is $0.2 \mathrm{~kJ} \mathrm{~mol}^{-1}$ per CuF higher in energy in comparison to cinnabar-type CuF.

\section{CuF structures from USPEX simulation with composition $\mathrm{Cu}_{8} \mathrm{~F}_{8}$}

Finally, we ran one USPEX simulation with the composition $\mathrm{Cu}_{8} \mathrm{~F}_{8}$, producing 313 structures in total. Only four structures were found to possess lower energy than cinnabar-type CuF (Table 1). The USPEX simulations with $Z=8$ are already computationally rather demanding with the DFT-PBE0-D3 method and the small number of low-energy structures suggests that the configurational space has been rather wellspanned already by the USPEX simulations with smaller $Z$. The structures $\mathbf{Z 8 a} \mathbf{Z} \mathbf{Z 8 d}$ have lower relative energy than the lowest-energy $\mathbf{Z 2}$ structures, but overall the structure $\mathbf{Z 8 a}$ is not among the 10 lowest energy structures $\left(\Delta E=-2.5 \mathrm{~kJ} \mathrm{~mol}^{-1}\right.$ per $\mathrm{CuF}$ ). To summarize, the composition $\mathrm{Cu}_{2} \mathrm{~F}_{2}$ appears to be too small to span essential parts of the configurational space, while $\mathrm{Cu}_{8} \mathrm{~F}_{8}$ is already computationally rather demanding and does not produce low-energy structures with as good hit rate as the compositions $\mathrm{Cu}_{3} \mathrm{~F}_{3}-\mathrm{Cu}_{6} \mathrm{~F}_{6}$.

\section{Relative energies of the predicted CuF structures studied with the ab initio LMP2 method}

The DFT-PBE0-D3/TZVP energy comparisons show that there are three hypothetical CuF structures for which the relative energy is lower than $-4.0 \mathrm{~kJ} \mathrm{~mol}^{-1}$ per CuF (Z3a, Z4a, and Z5a with $\Delta E=-7.9,-4.9$, and $-4.3 \mathrm{~kJ} \mathrm{~mol}^{-1}$ per CuF unit, respectively). In addition, there are many structural candidates within a rather narrow energy range from -3 to $-4 \mathrm{~kJ} \mathrm{~mol}^{-1}$ per $\mathrm{CuF}$ unit. Even the lowest-energy structures found here do not appear to be stable with respect to disproportionation to $\mathrm{CuF}_{2}$ and $\mathrm{Cu}$ metal at ambient pressure. The disproportionation reaction $\operatorname{CuF}(\mathrm{Z3a}) \rightarrow \mathrm{CuF}_{2}+\mathrm{Cu}$ is exoenergetic by $-37 \mathrm{~kJ}$ $\mathrm{mol}^{-1}$, whereas previous studies reported $-34 \mathrm{~kJ} \mathrm{~mol}^{-1}$ and -49 $\mathrm{kJ} \mathrm{mol}^{-1}{ }^{11,16}$ We also investigated the disproportionation reaction at $10 \mathrm{GPa}$ pressure, where the reaction is still exoenergetic by $-37 \mathrm{~kJ} \mathrm{~mol}^{-1}$.

To further investigate the energetics of the predicted CuF structures, in particular from the point of view of the weak $\mathrm{Cu}-$ $\mathrm{Cu}$ interactions, we also carried out single-point energy calculations at the LMP2/TZVPP level of theory (Table 2). ${ }^{64}$ While the D3 dispersion correction is empirical, the LMP2 method offers an ab initio approach for evaluating the energetics of the $\mathrm{CuF}$ structures with weak $\mathrm{Cu}-\mathrm{Cu}$ interactions.
Table 2. Relative energies of the predicted CuF at the DFT-PBE0-D3/TZVP and LMP2/TZVPP levels of theory.

\begin{tabular}{|c|c|c|}
\hline \multirow[t]{2}{*}{ Structure } & \multicolumn{2}{|c|}{$\Delta E\left(\mathrm{~kJ} \mathrm{~mol}^{-1}\right.$ per CuF unit) } \\
\hline & $\begin{array}{l}\text { DFT-PBE0- } \\
\text { D3/TZVP }\end{array}$ & LMP2/TZVPP \\
\hline Z3a & -7.9 & $-4.8(-5.4)^{\mathrm{a}}$ \\
\hline Z4a & -4.8 & $-7.0(-4.6)$ \\
\hline Z5a & -4.3 & $-1.9(-0.4)$ \\
\hline Z6a & -3.9 & $-3.8(-3.4)$ \\
\hline Z4b & -3.9 & $-0.8(-0.3)$ \\
\hline Z5b & -3.4 & 0.2 \\
\hline Z6b & -3.3 & 0.5 \\
\hline Z4c & -3.3 & -3.2 \\
\hline Z5c & -3.3 & $1.4(1.9)$ \\
\hline Z6c & -3.1 & $-5.6(-3.9)$ \\
\hline Z4d & -3.1 & -3.0 \\
\hline Z8a & -2.5 & 1.7 \\
\hline Z6d & -2.3 & $-4.4(-2.1)$ \\
\hline Z6e & -2.1 & -0.4 \\
\hline Z6f & -2.0 & 3.2 \\
\hline $\mathrm{Z6g}$ & -2.0 & -1.0 \\
\hline Z8b & -1.8 & -0.5 \\
\hline Z6h & -1.7 & 2.8 \\
\hline Z6i & -1.7 & -1.3 \\
\hline Z4e & -1.7 & 1.2 \\
\hline Z4f & -1.6 & -0.5 \\
\hline Z6j & -1.4 & 1.9 \\
\hline $\mathrm{Z4g}$ & -1.4 & 0.4 \\
\hline $\mathrm{Z4h}$ & -1.3 & 1.5 \\
\hline $\mathrm{Z4i}$ & -1.3 & -1.5 \\
\hline Z8c & -1.0 & 0.7 \\
\hline $\mathrm{Z} 4 \mathrm{j}$ & -0.9 & 2.2 \\
\hline Z4k & -0.8 & 2.1 \\
\hline Z6k & -0.6 & -0.4 \\
\hline Z3b & -0.6 & 3.5 \\
\hline Z3c & -0.4 & 3.6 \\
\hline Z4I & -0.4 & 3.3 \\
\hline Z8d & -0.3 & 2.9 \\
\hline $\mathrm{Z} 4 \mathrm{~m}$ & -0.3 & -2.8 \\
\hline $\mathrm{Z} 4 \mathrm{n}$ & -0.2 & 2.7 \\
\hline Z4o & -0.2 & -0.6 \\
\hline $\mathrm{Z4p}$ & -0.1 & 1.9 \\
\hline $\mathrm{Z2a}$ & -0.1 & 2.5 \\
\hline $\mathrm{Z2b}$ & -0.1 & -0.7 \\
\hline
\end{tabular}

a Relative energies in parentheses have been obtained with the extended $T Z V P P+f$ basis set (see text).

The DFT-PBE0-D3/TZVP and LMP2/TZVPP relative energies of the hypothetical CuF structures are illustrated in Figure 8. Altogether 39 crystal structures were predicted by DFT to have lower energy than the cinnabar-type CuF. Single-point energy calculations of these structures with LMP2 suggest that 19 of the structures have lower energy than cinnabar-type CuF.

The two lowest-energy structures predicted by DFT retain a low relative energy also with LMP2, but their order is reversed. The relative energy of the structure Z3a deteriorates from -7.9 to $-4.8 \mathrm{~kJ} \mathrm{~mol}^{-1}$ per CuF unit, whereas the relative energy of the structure Z4a improves from -4.9 to $-7.0 \mathrm{~kJ} \mathrm{~mol}^{-1}$ per CuF unit. The relative energy of $\mathbf{Z 5 a}$ deteriorates from -4.3 to $-1.9 \mathrm{~kJ} \mathrm{~mol}^{-}$ 1 per CuF unit.

Concerning the DFT and LMP2 relative energies of other low-energy structures, the two methods are in relatively good agreement for the following cases (energies in $\mathrm{kJ} \mathrm{mol}^{-1}$ per CuF unit): -3.3 vs. -3.2 for $\mathbf{Z} \mathbf{4 c},-3.1$ vs. -3.0 for $\mathbf{Z} 4 \mathbf{d},-1.3$ vs. -1.5 for $\mathbf{Z 4 i},-0.2$ vs. -0.6 for $\mathbf{Z 4 o},-3.9$ vs. -3.8 for $\mathbf{Z 6 a},-1.7$ vs. - 
1.3 for $\mathbf{Z 6 i},-0.6$ vs. -0.4 for $\mathbf{Z 6 k}$. In some cases, though, the DFT and LMP2 energies differed significantly. The largest deviations between the DFT and LMP2 energies were found to be about $5 \mathrm{~kJ} \mathrm{~mol}^{-1}\left(-3.3 \mathrm{~kJ}\right.$ vs. $1.4 \mathrm{~kJ} \mathrm{~mol}^{-1}$ per CuF unit for $\mathbf{Z 5 c},-2.0 \mathrm{~kJ}$ vs. $3.2 \mathrm{~kJ} \mathrm{~mol}^{-1}$ per CuF unit for Z6f, $-1.7 \mathrm{~kJ}$ vs. 2.8 $\mathrm{kJ} \mathrm{mol}^{-1}$ per CuF unit for Z6h).

It appears that the CuF structures where the shorter $\mathrm{Cu}-\mathrm{F}$ distances are larger than $1.96 \AA$ become energetically less favorable with LMP2/TZVPP (structures Z2a, Z3a, Z3b, Z3c, Z4b, Z4e-Z4h, Z4j-Z4I, Z4n, Z5a, Z4p, Z5b, Z5c, Z6b, Z6eZ6h, Z6j, Z8a, Z8c, Z8d). For comparison, CuF structures where the shorter $\mathrm{Cu}-\mathrm{F}$ distances are less than $1.96 \AA$ appear to have similar relative energies with DFT-PBE0-D3/TZVP and LMP2/TZVPP (Z4o, Z8b) or possess even lower relative energy with LMP2 (Z2b, Z4a, Z4m, Z6c, Z6d). Since all the structures have been obtained as a result of DFT structural optimizations, it can be that not all of the structures are energetically that favorable with LMP2. USPEX simulations with LMP2 are not yet feasible since analytical LMP2 gradients are not available and the HF+LMP2 calculations are significantly more demanding computationally compared to hybrid DFT.

The LMP2/TZVPP single-point energies also highlighted two structures, where the relative energy improved so much that they became low-energy structures (Z6c with improvement from -3.1 to $-5.6 \mathrm{~kJ} \mathrm{~mol}^{-1}$ per CuF unit and $\mathbf{Z 6 d}$ with improvement from -2.3 to $-4.4 \mathrm{~kJ} \mathrm{~mol}^{-1}$ per CuF unit). Thus, $\mathbf{Z 6 c}$ is the structure with the second-lowest relative energy at the LMP2/TZVPP level of theory.

For the five lowest-energy structures found by DFT-D3 (Z3a, Z4a, Z5a, Z6a, Z4b), as well as low-energy LMP2/TZVPP structures Z6c, and Z6d, we carried out LMP2 calculations with extended TZVPP $+f$ basis set to get a better understanding of the basis set effects (see Computational details). In these calculations, the relative LMP2 energy of Z3a changed from -4.8 to $-5.4 \mathrm{~kJ} \mathrm{~mol}^{-1}$ per CuF unit and the relative energy of $\mathbf{Z 4 a}$ changed from -7.0 to $-4.6 \mathrm{~kJ} \mathrm{~mol}^{-1}$ per CuF unit. This energy ordering of $\mathbf{Z 3 a}$ and $\mathbf{Z 4 a}$ is in agreement with the DFT-PBEOD3/TZVP results, but one should keep in mind that the geometries were not optimized with the LMP2 method and this could further change the relative ordering. The main finding is that the structures $\mathbf{Z 3} \mathbf{a}$ and $\mathbf{Z 4 a}$ are the lowest-energy structures at both DFT-PBE0-D3/TZVP and LMP2/TZVPP $+f$ levels of theory. For the three other structures $\mathbf{Z 5 a}, \mathbf{Z 6 a}$, and $\mathbf{Z 4 b}$, the LMP2 relative energies change as follows: from -3.8 to $-3.4 \mathrm{~kJ}$ $\mathrm{mol}^{-1}$ per CuF unit (Z6a), from -1.9 to $-0.4 \mathrm{~kJ} \mathrm{~mol}^{-1}$ per CuF unit (Z5a), and from -0.8 to $0.3 \mathrm{~kJ} \mathrm{~mol}^{-1}$ per CuF unit (Z4b). In the case of $\mathbf{Z 6 c}$ and $\mathbf{Z} 6 \mathbf{d}$, the relative energies change from 5.6 to $-3.9 \mathrm{~kJ} \mathrm{~mol}^{-1}$ per CuF unit (Z6c) and from -4.4 to $-2.1 \mathrm{~kJ}$ $\mathrm{mol}^{-1}$ per CuF unit, bringing them close to the DFT-PBE0/D3 relative energies. We also carried out extended basis set calculation for $\mathbf{Z 5 c}$, which has the largest deviation between the DFT-D3 and the LMP2 results. The relative LMP2 energy of the Z5c structure changed from 1.4 to $1.9 \mathrm{~kJ} \mathrm{~mol}^{-1}$ per CuF unit (DFT-D3: $-3.3 \mathrm{~kJ} \mathrm{~mol}^{-1}$ per CuF unit).

\section{Conclusions}

We have carried out crystal structure predictions for copper(I) fluoride by using the evolutionary algorithm-based USPEX code and a recently developed CRYSTAL interface. We screened about 5000 hypothetical crystal structures by using a dispersioncorrected hybrid DFT method and also carried out single-point energy calculations with ab initio LMP2 method. We identified 39 hypothetical CuF structures that are lower in energy in comparison to the previously reported cinnabar-type CuF structure (19 structures at the LMP/TZVPP level of theory). The predicted low-energy structures are true local minima that could exist as metastable species, but this remains to be proven by definitive experimental validation. Relatively short $\mathrm{Cu}-\mathrm{Cu}$ distances suggesting cuprophilic $\mathrm{Cu}-\mathrm{Cu}$ interactions are present in all the low-energy structures, leading in ordered $\mathrm{Cu}$ substructures. Minor structural modifications can lead to significant changes in the relative energies of the CuF structures. The lowest-energy CuF structures predicted here can be used to identify unknown phases in the experimental investigations towards copper(I) fluoride.

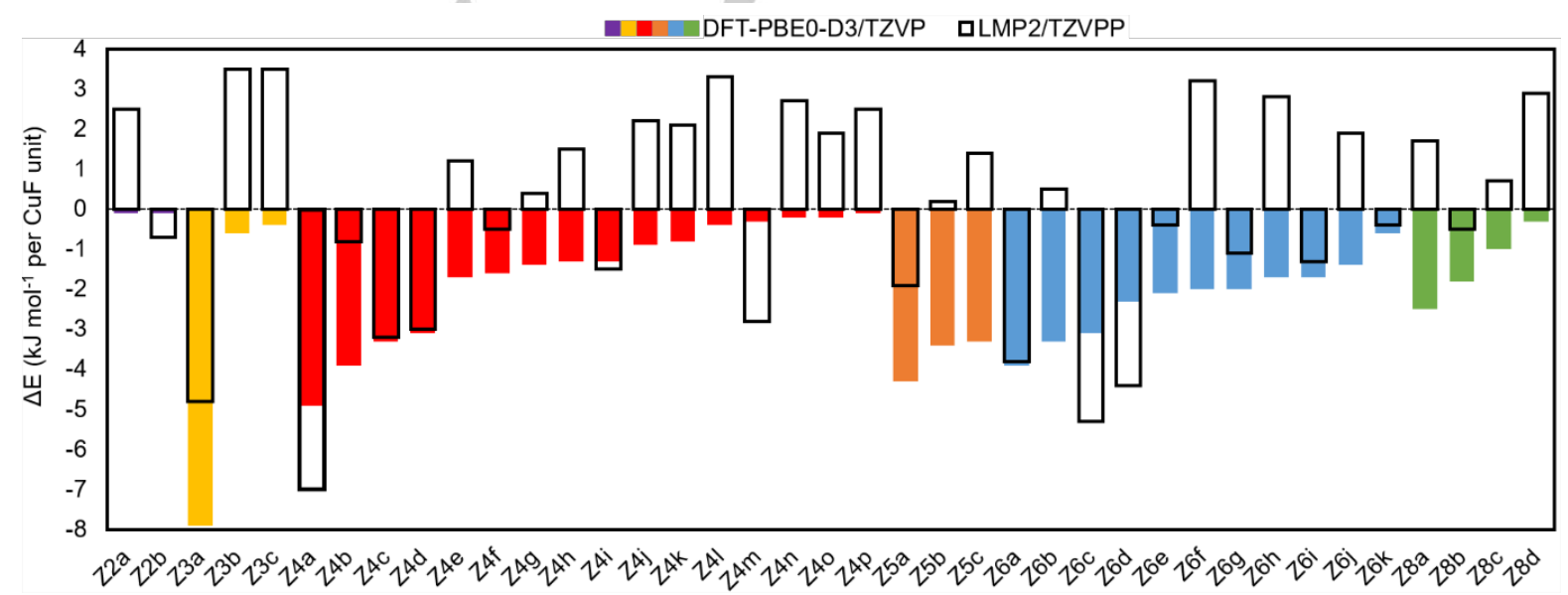

Figure 8. Relative DFT-PBE0-D3/TZVP and LMP2/TZVPP energies of the lowest-energy CuF structures predicted by USPEX. The energies are given with respect to the cinnabar-type $\operatorname{CuF}(\Delta E=0)$. For the labeling of the structures, please see the caption of Figure 2. 


\section{Computational details}

Crystal structure predictions were carried out by using USPEX 9.4 .4 code (USPEX input file is included as Supporting Information, SI). ${ }^{25,26,28}$ All quantum chemical calculations within the USPEX simulations were performed using the CRYSTAL17 code. ${ }^{34}$ We used a recently developed CRYSTAL interface for USPEX. ${ }^{27}$ Hybrid DFT-PBE0 functional with $25 \%$ Hartree-Fock exchange was utilized. ${ }^{35,36}$ For a $3 d$ metal such as $\mathrm{Cu}$, the use of hybrid DFT over GGA or GGA + $U$ is expected to increase the accuracy of the predictions. ${ }^{37-43}$ All-electron, Gaussian-type triple- $\zeta$ valence + polarization (TZVP) and split-valence + polarization (SVP) basis sets based on Karlsruhe def2 basis sets were used within the crystal structure predictions (a list with of all used basis sets is provided as $\mathrm{SI}) .{ }^{44} \mathrm{Cu}(\mathrm{I})$ ions are expected to show weak "cuprophilic" $d^{10}-d^{10}$ interactions, ${ }^{45-48}$ which were taken into account in the USPEX search using Grimme's D3 dispersion correction with zero-damping (ZD). ${ }^{49,50}$ To accelerate the evolutionary searches, the local geometry optimizations within USPEX were carried out by using relatively weak convergence criteria and, in some cases, the smaller SVP basis set. Input files for CRYSTAL geometry optimizations within the USPEX search are provided as SI.

Low-energy structures produced by USPEX were re-optimized at the DFT-PBE0-D3(ZD)/TZVP level of theory using tighter convergence criteria. For the re-optimization, the reciprocal space $k$-point meshes were chosen depending on the magnitude of the corresponding direct space lattice parameter $d$ : $d<4 \AA \rightarrow 12 k$-points along $d ; 4 \AA<d<6 \AA$ $\rightarrow 8,6 \AA<d<8 \AA \rightarrow 6 ; 8 \AA<d<12 \AA \rightarrow 4, d>12 \AA \rightarrow 2$. Tightened tolerance factors (TOLINTEG) of $8,8,8,8$, and 16 were used for the evaluation of the Coulomb and exchange integrals. All re-optimized structures that were found to be at least $3 \mathrm{~kJ} \mathrm{~mol}^{-1}$ per CuF lower in energy compared to the cinnabar-type structure were confirmed to be true local minima by means of a harmonic frequency calculation. ${ }^{51,52}$

To evaluate the performance of the applied USPEX/DFT-PBE0-D3(ZD) crystal structure prediction methodology for $d^{10}$-metal monofluorides, we confirmed that rocksalt structure $(F m-3 m)$ was correctly found as the lowest-energy structure for the known AgF. ${ }^{53}$

For investigating the energetics of the disproportionation reaction $\mathrm{CuF} \rightarrow$ $\mathrm{CuF}_{2}+\mathrm{Cu}$, we also optimized the structures of $\mathrm{CuF}_{2}$ and $\mathrm{Cu}$ at the DFTPBE0-D3/TZVP level of theory. $\mathrm{CuF}_{2}$ was studied using an antiferromagnetic ground state. ${ }^{54} 12 \times 8 \times 8$ and $16 \times 16 \times 16 k$-point meshes were used for $\mathrm{CuF}_{2}$ and $\mathrm{Cu}$ metal, respectively. For metallic $\mathrm{Cu}$, Fermi surface smearing with $T=316 \mathrm{~K}(0.001$ a.u. $)$ was applied and a doubledensity $k$-mesh was used for the determination of the Fermi energy. The optimized structures of $\mathrm{CuF}_{2}$ and $\mathrm{Cu}$ metal are included as SI. In the USPEX simulations on CuF, we do not expect to see any disproportionation to $\mathrm{CuF}_{2}$ and $\mathrm{Cu}$ as the used unit cells are still relatively small (up to $Z=8$ ) and we are running closed-shell calculations with no unpaired electrons.

To confirm the presence of weak $\mathrm{Cu}-\mathrm{Cu}$ interactions, we also carried out periodic local second-order Møller-Plesset perturbation theory (LMP2) single-point energy calculations for the CuF structures which were found to possess lower energy than the previously reported cinnabar-type structure at the DFT-PBE0-D3(ZD)/TZVP level of theory. The LMP2 calculations were carried out with a development version of the CRYSCOR software ${ }^{55}$ which implements orbital specific virtuals (OSVs) to represent the truncated pair-specific virtual space. ${ }^{56}$ In the OSV-LMP2 formalism, it is not necessary to manually define excitation domains for the virtual space as in the previous implementation based on projected atomic orbitals (PAO-LMP2). The OSV-LMP2 straightforwardly enables the calculation of smooth potential energy surfaces and relative energies of structural frameworks with different topologies. ${ }^{57}$

The HF reference wavefunction and the localized valence-space Wannier functions (WFs) necessary for the LMP2 procedure were obtained with CRYSTAL17. Very tight TOLINTEG tolerance factors of $10,10,10,20$ and 50 were used in the Hartree-Fock (HF) part. All-electron, and triple- $\zeta$ valence + double polarization (TZVPP) basis set based on Karlsruhe def2-TZVPP basis set was used for $\mathrm{Cu}$, while TZVP basis set was used for F. For the five CuF structures with the lowest energy according to DFT-D3, we also calculated LMP2 single-point energies using even larger basis set that added an f-function for $\mathrm{F}$ and a third f-function for $\mathrm{Cu}$. These calculations were carried our using a dual basis set scheme. ${ }^{58,59}$ In the LMP2 calculations, we utilized the direct-space density-fitting technique for computing the two-electron four-index integrals. A Poisson/Gaussian-type auxiliary basis set of triple-zeta-valence quality was employed for the density-fitting. ${ }^{60-62}$ From a practical point of view, the calculation of the reference wavefunction with $\mathrm{HF}$ can be computationally even more expensive than the actual LMP2 calculation. An input file example for the CRYSCOR calculations is available as SI.

\section{Acknowledgements}

Funding from the Academy of Finland (grant no. 317273) and computing resources from CSC, the Finnish IT Center for Science, are gratefully acknowledged. F. K. thanks the Deutsche Forschungsgemeinschaft (DFG) for generous funding.

\section{Keywords: Copper • Fluorides • Structure elucidation •}

Semiconductors $\cdot$ Density functional calculations

S. Chen, X. G. Gong, A. Walsh and S. H. Wei, Electronic structure and stability of quaternary chalcogenide semiconductors derived from cation cross-substitution of II-VI and I-III-VI2 compounds, Phys. Rev. B, 2009 79, 1-10.

J. J. Berzelius, Untersuchungen uber die flussspathsssaure und deren merkwurdigsten Verbindungen, Ann. Phys., 1824, 78, 113-150.

$\mathrm{H}$. Woitinek and F. Ebert, Kristallstrukturen von fluoridem. II. $\mathrm{HgF}, \mathrm{HgF}_{2}$ CuF and $\mathrm{CuF}_{2}$, Z. Anorg. Allg. Chem., 1933, 78, 269-272.

4 V. O. Ruff and M. Giese, Die fluorierung des silbers und kupfers, $Z$ Anorg. Allg. Chem., 1934, 219, 143-148.

5 H. von Wartenberg, Uber kupferfluoride, Z. Anorg. Allg. Chem., 1939, 241, 381-394

6 W. Klemm and E. Huss, Fluorokomplexe. I. Eisen-, kobalt-, nickel- und kupfer-komplexe, Z. Anorg. Allg. Chem., 1949, 258, 221-226.

7 J. M. Crabtree, C. S. Lees and K. Little, The copper fluorides. Part I - XRay and electron microscope examination, J. Inorg. Nucl. Chem., 1955, 1, 213-217.

8 C. Poulenc, Etude des fluorures de chrome, Compt. Rend., 1893, 116 1446-1449.

9 C. Poulenc, Fluorures de cuivre, Ann. Chim. Phys., 1894, 7, 68-73.

10 H. M. Haendler, L. H. Towle, E. F. Benntt and W. L. Patterson, The Reaction of fluorine with copper and some of its compounds. Some properties of copper(II) fluoride, J. Am. Chem. Soc., 1954, 76, 21782179.

11 P. Woidy, A. J. Karttunen, M. Widenmeyer, R. Niewa and F. Kraus, On copper(I) fluorides, the cuprophilic interaction, the preparation of copper nitride at room temperature, and the formation mechanism at elevated temperatures, Chem.: Eur. J., 2015, 21, 3290-3303. 
12 Z. Mazej and P. Benkic, Copper(I) hexafluoroantimonate - an example of a compound with $\mathrm{Cul}$ in a solely fluorine environment, J. Fluorine. Chem., 2005, 126, 803-808.

13 M. Barber and T. N. H. Linnett, The Halides of the transition elements of the first long period, J. Chem. Soc., 1961, 3323-3332.

14 T. Sohnel, H. Hermann and P. Schwerdtfeger, Solid state density functional calculations for the group 11 monohalides, J. Phys. Chem. B, 2005, 109, 526-531.

15 R. P. Krawczyk, A. Hammerl and P. Schwerdtfeger, Coinage meta halide clusters: From two-dimensional ring to structures, ChemPhysChem, 2006, 7, 2286-2289.

16 A. Walsh, C. R. A. Catlow, R. Galvelis, D. O. Scanlon, F. Schiffmann, A. A. Sokol and S. M. Woodley, Prediction on the existence and chemical stability of cuprous fluoride, Chem. Sci., 2012, 3, 2565-2569.

17 J. C. Schön and M. Jansen, First step towards planning of syntheses in solid-state chemistry: Determination of promising structure candidates by global optimization, Angew. Chem. Int. Ed., 1996, 35, 1286-1304.

18 S. E. Schönborn, S. Goedecker, S. Roy and A. R. Oganov, The performance of minima hopping and evolutionary algorithms for cluster structure prediction, J. Chem. Phys., 2009, 130, 1-9.

19 R. Martoňák, A. Laio and M. Parrinello, Predicting crystal structures: The Parrinello-Rahman method revisited, Phys. Rev. Lett., 2003, 90 075503.

20 S. Goedecker, Minima hopping: An efficient search method for the global minimum of the potential energy surface of complex molecular systems, J. Chem. Phys., 2004, 120, 9911-9917.

21 M. P. R. Martoňák, A. Laio, M. Bernasconi, C. Ceriani, P. Raiteri, F. Zipoli, Simulation of structural phase transitions by metadynamics, Zeitschrift für Krist., 2005, 220, 489-498.

22 S. M. Woodley, P. D. Battle, J. D. Gale and R. A. C. Catlow, The prediction of inorganic crystal structures using a genetic algorithm and energy minimisation, Phys. Chem. Chem. Phys., 1999, 1, 2535-2542.

23 S. M. Woodley, Prediction of crystal structures using evolutionary algorithms and related techniques, Struct. Bond., 2004, 110, 95-132.

24 A. R. Oganov and C. W. Glass, Crystal structure prediction using ab initio evolutionary techniques: Principles and applications, J. Chem. Phys., 2006, 124, 1-15.

25 A. R. Oganov, A. O. Lyakhov and M. Valle, How evolutionary crystal structure prediction works-and why, Acc. Chem. Res., 2011, 44, 227 237.

26 A. O. Lyakhov, A. R. Oganov, H. T. Stokes and Q. Zhu, New developments in evolutionary structure prediction algorithm USPEX, Comput. Phys. Commun., 2013, 184, 1172-1182.

27 M. S. Kuklin and A. J. Karttunen, Crystal structure prediction of magnetic transition metal oxides by using evolutionary algorithm and hybrid DFT methods, J. Phys. Chem. C, 2018, 122, 24949-24957.

28 C. W. Glass, A. R. Oganov and N. Hansen, USPEX-Evolutionary crystal structure prediction, Comput. Phys. Commun., 2006, 175, 713720

29 A. R. Oganov, Y. Ma, C. W. Glass and M. Valle, Evolutionary crystal structure prediction: overview of the USPEX method and some of its applications, Psi-k Newsl., 2007, 84, 142-171.

30 A. R. Oganov and C. W. Glass, Evolutionary crystal structure prediction as a tool in materials design, J. Phys. Condens. Matter, 2008, 20 064210 .

31 A. R. Oganov and M. Valle, How to quantify energy landscapes of solids, J. Chem. Phys., 2009, 130, 2-10.

32 A. O. Lyakhov, A. R. Oganov and M. Valle, How to predict very large and complex crystal structures, Comput. Phys. Commun., 2010, 181, 1623-1632.

33 A. R. Oganov, Modern methods of crystal structure prediction, Wiley$\mathrm{VCH}$, Berlin, 2010.

34 R. Dovesi, A. Erba, R. Orlando, C. M. Zicovich-Wilson, B. Civalleri, L. Maschio, M. Rérat, S. Casassa, J. Baima, S. Salustro and B. Kirtman,
Quantum-mechanical condensed matter simulations with CRYSTAL, Wiley Interdiscip. Rev. Comput. Mol. Sci., 2018, 8, 1-36.

35 J. P. Perdew, K. Burke and M. Ernzerhof, Generalized gradien approximation made simple, Phys. Rev. Lett., 1996, 77, 3865-3868.

36 C. Adamo and V. Barone, Toward reliable density functional methods without adjustable parameters: The PBE0 model, J. Chem. Phys., 1999, 110, 6158-6170.

37 J. Linnera and A. J. Karttunen, Ab initio study of the lattice thermal conductivity of $\mathrm{Cu}_{2} \mathrm{O}$ using the generalized gradient approximation and hybrid density functional methods, Phys. Rev. B, 2017, 96, 014304

38 L. Wang, T. Maxisch and G. Ceder, Oxidation energies of transition metal oxides within the GGA+U framework, Phys. Rev. B, 2006, 73, 195107.

39 C. Rödl, F. Fuchs, J. Furthmüller and F. Bechstedt, Quasiparticle band structures of the antiferromagnetic transition-metal oxides $\mathrm{MnO}, \mathrm{FeO}$ CoO, and NiO, Phys. Rev. B, 2009, 79, 235114.

40 H. J. Kulik and N. Marzari, Transition-metal dioxides: A case for the intersite term in Hubbard-model functionals, J. Chem. Phys., 2011, 134, 094103.

41 X. Chen, D. Parker, M. H. Du and D. J. Singh, Potential thermoelectric performance of hole-doped $\mathrm{Cu}_{2} \mathrm{O}$, New J. Phys., 2013, 15, 043029.

42 D. H. Seo, A. Urban and G. Ceder, Calibrating transition-metal energy levels and oxygen bands in first-principles calculations: Accurate prediction of redox potentials and charge transfer in lithium transitionmetal oxides, Phys. Rev. B, 2015, 92, 115118.

43 R. Gillen and J. Robertson, Accurate screened exchange band structures for the transition metal monoxides $\mathrm{MnO}, \mathrm{FeO}, \mathrm{CoO}$ and $\mathrm{NiO}$ J. Phys. Condens. Matter, 2013, 25, 165502.

44 F. Weigend and R. Ahlrichs, Balanced basis sets of split valence, triple zeta valence and quadruple zeta valence quality for $\mathrm{H}$ to $\mathrm{Rn}$ : Design and assessment of accuracy, Phys. Chem. Chem. Phys., 2005, 7 , 3297-3305.

45 P. Pyykko, N. Runeberg and F. Mendizabal, Theory of the $d^{10}-d^{10}$ closed-shell attraction: 1. Dimers near equilibrium, Chem. Soc. Rev., 1997, 3, 1451-1457.

46 H. L. Hermann, G. Boche and P. Schwerdtfeger, Metallophilic interactions in closed-shell copper(I) compounds - A theoretical study, Chem.: Eur. J., 2001, 7, 5333-5342.

47 E. O. Grady and N. Kaltsoyannis, Does metallophilicity increase or decrease down group 11? Computational investigations of [Cl-M-PH 3$]$ (M = Cu, Ag, Au, [111]), Phys. Chem. Chem. Phys., 2004, 6, 680-687.

48 M. Angels Carvajal, S. Alvarez and J. J. Novoa, The nature of intermolecular Cul...Cul Interactions: A combined theoretical and structural database analysis, Chem.: Eur. J., 2004, 10, 2117-2132.

49 S. Grimme, J. Antony, S. Ehrlich and H. Krieg, A consistent and accurate ab initio parametrization of density functional dispersion correction (DFT-D) for the 94 elements H-Pu, J. Chem. Phys., 2010, 132, 154104.

50 S. Grimme, A. Hansen, J. G. Brandenburg and C. Bannwarth, Dispersion-corrected mean-field electronic structure methods, Chem. Rev., 2016, 116, 5105-5154.

51 C. M. Zicovich-Wilson, F. Pascale, C. Roetti, V. R. Saunders, R Orlando and R. Dovesi, Calculation of the vibration frequencies of aquartz : the effect of hamiltonian and basis set, J. Comput. Chem., 2004, 25, 1873-1881.

52 F. Pascale, C. M. Zicovich-Wilson, F. Lopez Gejo, B. Civalleri, R. Orlando and R. Dovesi, The calculation of the vibrational frequencies of crystalline compounds and its implementation in the CRYSTAL code, $J$. Comput. Chem., 2004, 25, 888-897.

$53 \mathrm{H}$. Ott, XI. Die Strukturen von $\mathrm{MnO}, \mathrm{MnS}, \mathrm{AgF}, \mathrm{NiS}, \mathrm{SnJ}_{4}, \mathrm{SrCl}_{2}, \mathrm{BaF}_{2}$; Präzisionsmessungen einiger Alkalihalogenide, Z. Krist., 1926, 63, 220-230.

54 P. Fischer, W. Halg, D. Schwarzenbach and H. Gamsjäger, Magnetic and crystal structure of copper(II) fluoride, J. Phys. Chem. Solids, 1974, 35, 1683-1689. 
C. Pisani, M. Schütz, S. Casassa, D. Usvyat, L. Maschio, M. Lorenz and A. Erba, CRYSCOR: a program for the post-Hartree-Fock treatment of periodic systems, Phys. Chem. Chem. Phys., 2012, 76157628.

56 D. Usvyat, L. Maschio and M. Schütz, Periodic local MP2 method employing orbital specific virtuals, J. Chem. Phys., 2015, 143, 102805.

57 A. J. Karttunen, D. Usvyat, M. Schitz and L. Maschio, Dispersion interactions in silicon allotropes, Phys. Chem. Chem. Phys., 2017, 19, 7699-7707.

58 K. Wolinski and P. Pulay, Second-order Møller-Plesset calculations with dual basis sets, J. Chem. Phys., 2006, 118, 9497.

59 D. Usvyat, L. Maschio, C. Pisani and M. Schütz, Second order local Møller-Plesset perturbation theory for periodic systems: the CRYSCOR code, Z. Phys. Chem., 2010, 224, 441-454.
60 M. Schütz, D. Usvyat, M. Lorenz, C. Pisani, L. Maschio, S. Casassa and M. Halo, in CRC Press, ed. Frederick R. Manby, Taylor and Francis, 2010, Accurate Condensed-Phase Quantum Chemsitry, 29-55. 61 L. Maschio, D. Usvyat, F. R. Manby, S. Casassa, C. Pisani and M. Schütz, Fast local-MP2 method with density-fitting for crystals . I. Theory and algorithms, Phys. Rev. B, 2007, 76, 075101.

62 L. Maschio and D. Usvyat, Fitting of local densities in periodic systems, Phys. Rev. B, 2008, 78, 073102

63 P. Pekka, Additive Covalent radii for single - , double - , and triplebonded molecules and tetrahedrally Bonded Crystals: A Summary, J. Phys. Chem. A, 2015, 119, 2326-2337.

64 D. Usvyat, L. Maschio and M. Schütz, Periodic and fragment models based on the local correlation approach, WIREs Comput. Mol. Sci., 2018, 8, 1-27. 


\section{Entry for the Table of Contents}

\section{FULL PAPER}

Evolutionary crystal structure prediction algorithms combined with dispersion-corrected hybrid density functional calculations yield a large number of new lowenergy structural candidates for the unknown copper(I) fluoride.

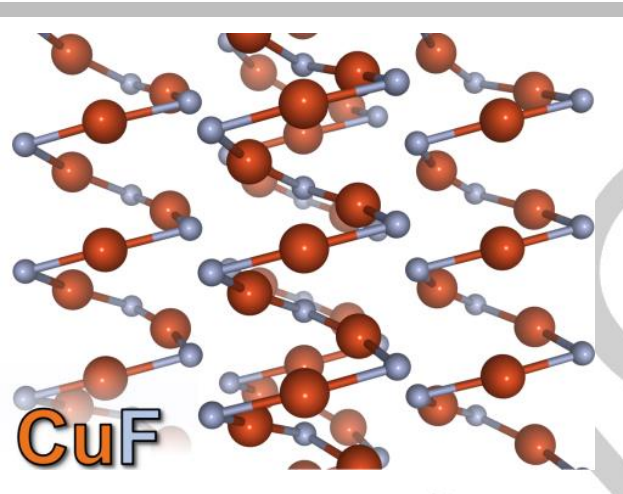

Mikhail S. Kuklin, Lorenzo Maschio, Denis Usvyat, Florian Kraus, Antti J. Karttunen*

\section{Evolutionary Algorithm-based} Crystal Structure Prediction for Copper(I) Fluoride 